\title{
Spreading of Low Impact Velocity Droplet on Porous Surface
}

\author{
Abdul Basit, KuZilati KuShaari, Thanh H. Trinh, and Babar Azeem
}

\begin{abstract}
Droplet spreading on flat non-reactive surfaces is established; however, porous surfaces present a complicated case. Wetting of porous surface involves the simultaneous spreading and penetration of the droplet. The effect of low impact velocity (i.e $<1 \mathrm{~m} / \mathrm{s}$ ) on dimensionless droplet diameter and dynamic contact angle has been experimentally observed in this study. Low impact velocity helps reducing the penetration of droplets into the droplets in case of porous substrates. In the low impact velocity range, the effect on contact angle is not significant. Dimensionless droplet diameter also shows similar behavior.
\end{abstract}

Index Terms-Contact angle, wetting, porous surface, droplet impact velocity.

\section{INTRODUCTION}

Droplet spreading has been studied for over a century [1]. The knowledge of droplet spreading is applied in spray coating, spray cooling and ink jet printing [2], [3]. The spreading behavior of droplet depends upon many factors like impact velocity, liquid characteristics (e.g. surface tension, viscosity and density) and the nature of the substrate. Small droplets are usually considered spherical if their capillary length is less than the diameter [4]. The spreading of droplet also depends upon whether the surface is dry or wet. Droplet impact is usually described by dimensionless numbers namely Reynolds number $\left(\frac{U D}{v}\right)$, Weber number

$\left(\frac{\rho U^{2} D}{\sigma}\right)$, Ohnesorge number and Capillary number, where $U$ is impact velocity, $D$ is droplet diameter, $v$ is kinematic viscosity of the liquid and $\sigma$ is surface tension. It is very important to be able to predict the maximum spreading factor (the ratio of spreading diameter to the initial diameter of the droplet) under given conditions. The advancement in this field transpired with the introduction of high speed cameras. Complicated spreading situations are yet not well understood [5].

Droplet spreading involves a wide range of values for the process variable involved [5]. Following is an up-close review solely for isothermal spreading with no phase transition.

\section{SPREADING ON NON-PoROUS SURFACE}

Most of the studies involve high impact velocities. For dry

Manuscript received August 3, 2013; revised November 25, 2013. This work was supported in part by the One Baja LRGs Grant.

The authors are with the Universiti Teknologi Petronas, Tronoh, Perak 31750, Malaysia (e-mail: abdulbasit135@gmail.com, kuzilati_kushaari@petronas.com.my, onalone2000@gmail.com, engrbabara@gmail.com). surfaces with high Reynolds number and high Weber number, the droplet usually forms a radially spreading lamella and a circular rim around it due to capillary forces and viscosity effects [6].

The droplet impact depends upon the impact parameters, properties of the liquid and also on the nature, topology and properties of the solid substrate. On dry surface some common outcomes of droplet impact may be droplet deposition, prompt splash, receding, partial or complete rebound [6]. Some new phenomena have also been observed like very thin gas layer in start of spreading, collision of two drops, bubble entrapment in spreading contacting line, lamella rupture in high velocity impacts, finger formation and periodic recoiling of droplet [6], [7].

Phenomenological details of droplet spreading dynamics on flat solid surface below splashing threshold/ Initial phase of droplet impact are as follows:

Rioboo et al. [8] described the droplet impact of Newtonian fluids on dry surfaces to be consisting of processes namely kinetic phase, spreading phase and relaxation phase. In the kinetic phase, liquid is compressed and shock wave is formed as shown in Fig. 1.

. Increasing impact velocity and droplet diameter results in faster spreading while increasing surface tension and viscosity leads to slower spreading [9], [10]. The results suggest that at high $\operatorname{Re}$ no $(>2000)$, and in the absence of splashing, Re no alone can represent the droplet deformation. This applies only if the surface roughness is very small in comparison to the film thickness. Otherwise film detachment will take place and hence splashing may occur. Increased roughness promotes splashing [7].

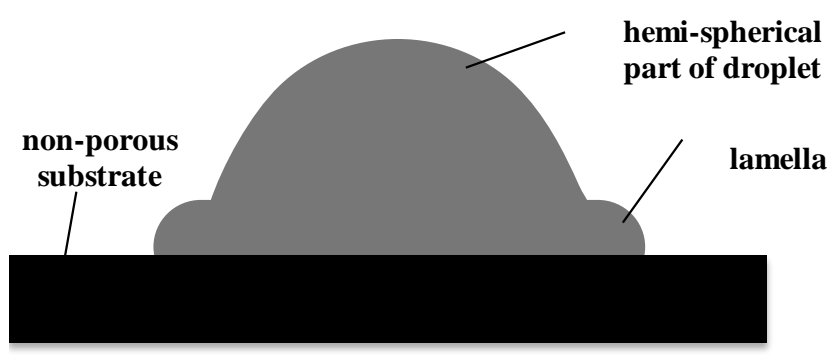

Fig. 1. Schematic of the initial stage of droplet impact

During the spreading phase, the hemi-sphere changes into lamella which thickens and expands to obtain disk like shape and sometimes has a rim around it as shown in Fig. 2.

The flow is directed from axial to radial direction. Generally, increasing the impact velocity and/or droplet diameter leads to faster spreading whereas increasing surface tension and/or viscosity results in slow expansion of droplet on the surface [8], [9], [11]. 


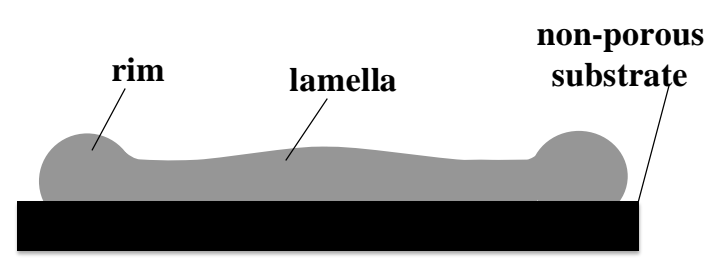

Fig. 2. Shape of droplet after a high velocity impact.

During the relaxation phase, droplet may start to recede. Wetting and surface tension are very important factors in this phase. The receding may end by reaching equilibrium if enough energy has been lost or another spreading phase may start if the surface is completely wetting.

Wettability is generally characterized by static (or equilibrium) contact angle [12] which is defined as "angle between tangent to the liquid interface and the tangent to the solid surface at the contact line between three phases" [13]. Usually contact angles are not unique for most of real surfaces. Wettability is not important in the first stage of spreading when the impact velocity is high because the droplet is spreading under inertia. Wettability only plays the role when the lamella has very slow velocity. Wettability and surface roughness are important parameters for spreading on flat dry surfaces [8].

\section{SPREAding On POROUS Surface}

Most studies relating to porous surfaces involve high impact velocities. High impact velocities result in more penetration than spreading. High penetration is not desirable in coating processes. Spreading on porous surfaces is a combination of two phenomena. First is the spreading of droplet over already saturated portions of the substrate which result in increase of droplet base. Second is the movement of droplet into the porous substrate which decreases droplet base however it increases depth of liquid wetted region inside the porous surface. Consequently the droplet radius attains a maximum value with time. Porous spreading studies require special attention during experiments. Because the results may be influenced by environmental effects e.g. temperature, humidity and vibrations [14].

Neyval et al. [15] studied the effect of seven important parameters for droplet spreading on porous surface including Reynolds no (Re), Darcy no (Da), Porosity $(\varepsilon)$, Froude no $(\mathrm{Fr})$, Weber no (We), contact angle $(\theta)$ and ratio between pore and particle diameter size in the porous substrate $(\alpha)$.

Capillarity is the driving force for the penetration of the fluids into the substrate [15], [16]. The penetration is characterized by a (constant) capillary suction acting across the wetting front, which may be regarded as measure for mean pore size [16].

Rapid deceleration of droplet in the impact region occurs thereby creating an increased pressure, and this elevated pressure is responsible for radial projection of liquid. Droplet spreads gradually but soon velocities start to decrease due to viscous effects [14], [15], [17]. At the same time, liquid penetrates into the porous substrate, though very slow as compared to lateral movement of fluid over the surface as shown in Fig. 3.

The dimensionless height of droplet above the surface decreases at a faster rate and simultaneously the droplet diameter expands rapidly. After some time, these changes slow down and ultimately stop. The liquid penetration, however, continues a little longer.

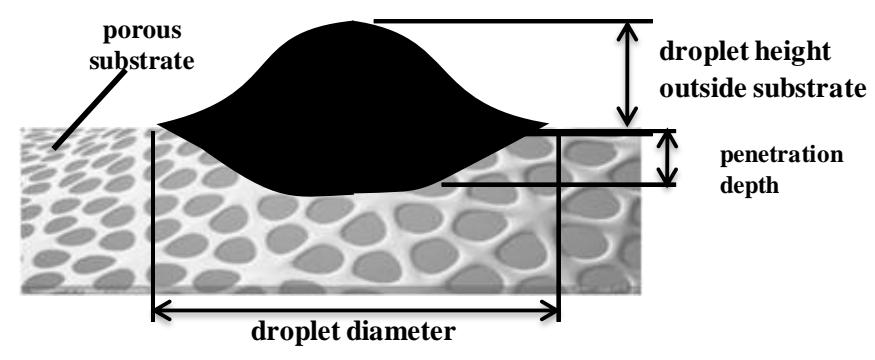

Fig. 3. Droplet spreading over porous surface.

\section{Methodology}

\section{A. Material Preparation}

The porous surface consists of flat urea surface. The flat urea surface was prepared by melting urea prills and allowing it to solidify into a plastic mould of cylindrical shape. The porosity of the urea surface was $21 \%$ as determined by He-Porosimeter.

The spreading liquid used was motor engine oil. The engine oil was used due to its inertness toward urea surface. The viscosity of the sample was reduced to 20 centi poise by adding benzene into it.

\section{B. Droplet Spreading Experiments}

Droplets of the oil were produced with the help of medical syringe. Droplet height was varied to study its effect on the droplet spreading parameters. The syringe height was varied to alter the droplet velocity. All other variables were kept constant. The droplet velocity was measured with the help of camera. The calibration of the camera was carried out for the droplet velocity determination. The droplet spreading was recorded with the help of Phantom high speed camera (Miro M320S). The experimental setup is shown in Fig. 4.

The light source was placed exactly opposite to the camera lens. The level of the camera was adjusted so that it is parallel to the solid surface.

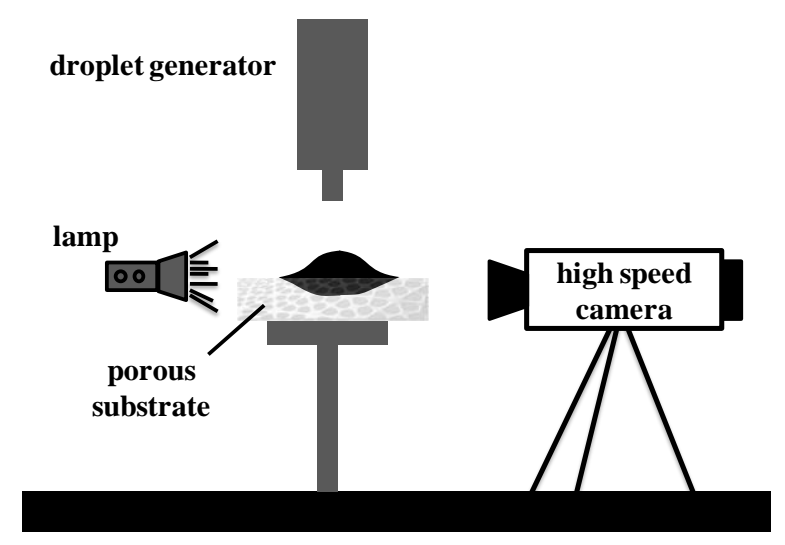

Fig. 4. Schematic of experimental setup to capture droplet spreading.

\section{Measurement of Droplet Dimensionless Diameter}

Droplet diameter was measured with the help of Phantom 
camera control application software $\mathrm{v} 2.14$. Value of droplet diameter at the time of its contact with the substrate is designated as $D_{\mathrm{o}}$. Time $t=0 \mathrm{sec}$ is also defined at this very moment. The diameter has been normalized using the initial droplet diameter i.e. $D=D_{\mathrm{o}} / D_{t}$ where $D$ is dimensionless diameter while Do is the initial diameter of the droplet and $D_{t}$ is diameter of the droplet at any given time $t$.

Diameter of the expanding droplet increases at a very fast rate for some time after hitting the substrate. Due to this reason, droplet diameter has been reported at $0.1 \mathrm{sec}$ interval for the first $1 \mathrm{sec}$ after the impact. For the next 10 seconds, the droplet diameter values have been reported at $1 \mathrm{sec}$ interval. The total duration of the spreading experiment reported is limited by the recording capacity of the camera.

\section{Contact Angle Measurement}

Droplet images were extracted from the recorded video at the required point of time. Edge detection algorithm namely 'Edge Hipass 3-by-3' was applied for edge detection to get a clear picture for contact angle measurement. Edge Hipass enhances 3-by-3 pixel translation areas or edges in an image, enhancing the high-frequency detail. Contact angle was measured using ImageJ software which utilizes low-bond axisymmetric drop shape analysis (LA-ADSA) method which is based on fitting of Young-Laplace equation to the image data [18].

\section{RESULTS AND DISCUSSION}

\section{A. Spreading of Droplet on Porous Surface}

Droplet spreading on porous surface is somewhat different than spreading on flat surface. In spreading on porous surface, the liquid penetrates and spreads at the same time. Either of these two processes (i.e. penetration and spreading) is faster than the other depending upon the porosity of the substrate, impact velocity of the droplet and the surface tension of the liquid [14]-[17], [19].
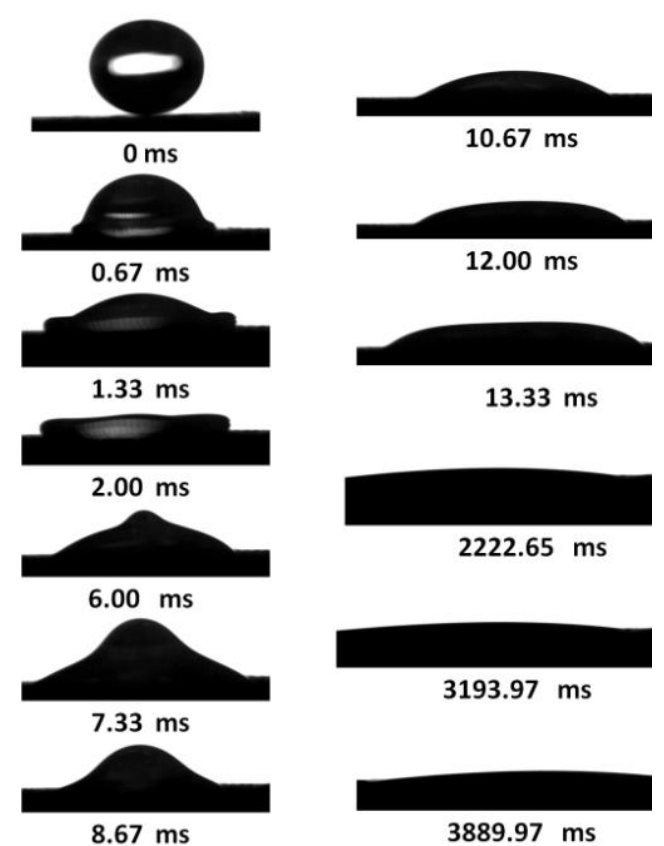

$13.33 \mathrm{~ms}$

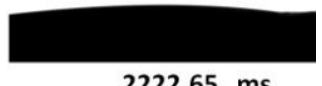

$2222.65 \mathrm{~ms}$

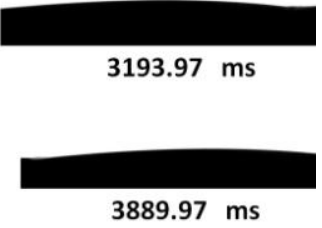

Fig. 5. Temporal variation of droplet shape after impact (time in milliseconds).
In the current study, the initial spreading of the droplets resembles that of the flat surface (Fig. 5). The droplet forms a lamella as it hits the surface which is usually observed during spreading on non-porous surfaces. This lamella can be seen in Fig. 5 at $0.67 \mathrm{~ms}$. Droplet also rebounds soon after hitting the surface. After this rebound, the droplet starts to spread in the lateral direction. The shape of the droplet at this stage resembles that of a pancake. Slowly the edges of the pancake become sharp and convert into the shape of hemisphere as seen at $10.67 \mathrm{~ms}$ in Fig. 5. The droplet continues its spreading and the penetration for a little longer. The changes in the shape of the droplet with the course of time are shown in Fig. 5

\section{B. Effect of Droplet Impact Velocity on Dimensionless Droplet Diameter}

The effect of droplet impact velocity on dimensionless droplet diameter has been experimentally studied using a defined porosity surface. In the current study, two different impact velocities have been studied. Spreading of sessile droplet has also been observed which serves as a base case.

Generally for any given impact velocity, the dimensionless droplet diameter increases rapidly as the droplet hits the substrate surface. As it can be seen in Fig. 6 that the dimensionless droplet diameter increases exponentially for the first 2 seconds. Kinetic energy of impact is being dissipated in lateral spreading of droplet. But the lateral expansion soon slows down and ultimately the value of droplet diameter becomes constant. It is also clear from the results that even in the narrow range of low impact velocities applied; the greater the impact velocity, greater is the dimensionless droplet diameter.

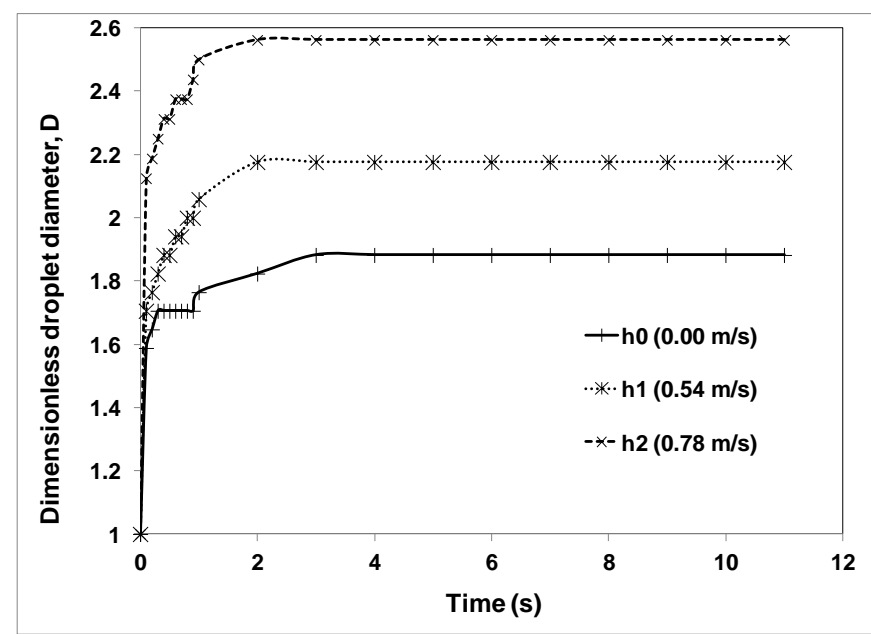

Fig. 6. Dimensionless droplet diameter vs time (s) for various impact velocities.

\section{Effect of Droplet Impact Velocity on Dynamic Contact Angle}

In case of spreading on porous surface, the decrease in contact angle is the combined effect of lateral movement of liquid above the surface as well as penetration into the substrate. The results show that contact angle decreases at a very fast rate at the start of the experiments until approximately 4 seconds. The rapid decrease in contact angle can be attributed to the lateral displacement of liquid above 
the substrate surface. Afterwards, contact angle still continues to decrease but at a slower pace. The fast rate of decrease in contact angle can be attributed to the lateral displacement of liquid above the substrate surface. While the second phase of slow rate of decrease in contact angle is due to the penetration of the liquid into the substrate structure which is a slow process. The phenomenon is also obvious form droplet dimensionless diameter measurements. Dimensionless diameter of the droplet changes at a fast rate similar to contact angle at the start of the experiments. But its value gets constant at almost the same time when the dynamic contact angle of the droplet was going through small changes at low pace. Value of diameter of droplet at this stage with slow change in contact angle value verifies the penetration of the liquid into the porous structure.

The dynamic contact angle depends on many factors including the droplet impact velocity, the surface tension of the liquid and wetting properties of the substrate. Effect of impact velocity on the contact angle has been analyzed while keeping the other parameters constant. Greater impact velocity facilitates the spreading and hence the contact angle decreases as the droplet spreads. However it is obvious that change in contact angle is not very significant at any given time due to the narrow range of impact velocities employed. The overlay of the variation in the contact angles is shown in Fig. 7. First curse marked as h0 represents the case of sessile drop. As the droplet hits the surface with some impact speed as compared to sessile drop, a marked difference in decrease of contact angle values is visible in the second curve marked as h1. Small increment in the impact velocity has little effect on contact angle value as shown in third curve marked as h2. So low impact speeds have little effect on contact angle.

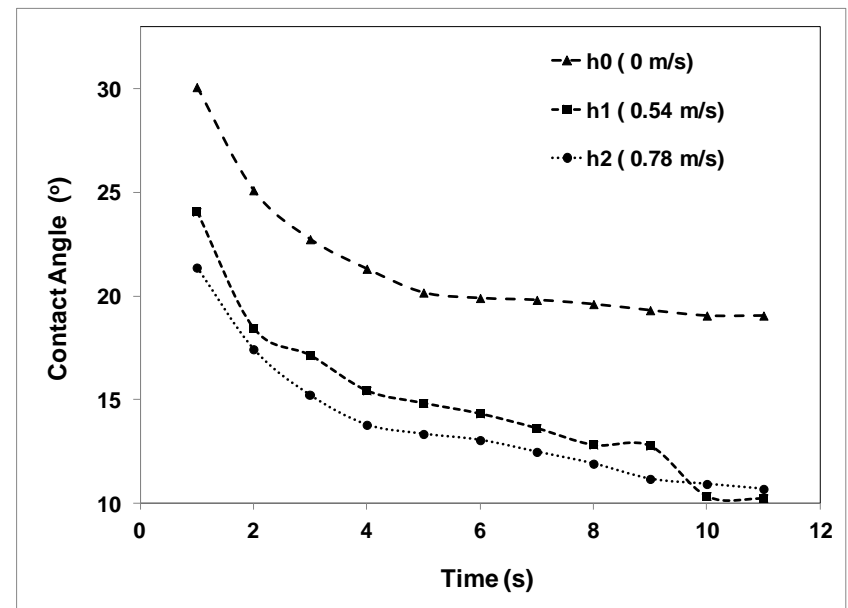

Fig. 7. Variation of contact angle (o) vs time (s) for various impact velocities.

\section{CONCLUSION}

The effect of droplet impact velocity on the dimensionless droplet diameter and dynamic contact angle has been studied. It has been found that the dimensionless droplet diameter increases at a rapid velocity in the start of the spreading process but subsequently slows down following a constant value. The dimensionless diameter increases as the impact velocity increases even in the narrow range of low impact velocities applied in this study.
The dynamic contact angle decreases as the spreading process continues. The dynamic contact angle also shows a sharp decrease at the start of the process owing to the expansion of the droplet above the surface. The subsequent decrease in the contact angle is due to the penetration of the liquid into the porous substrate.

The droplet spreading and penetration both are supported by the increasing impact velocities as observed for the different velocity ranges in this study.

\section{ACKNOWLEDGEMENT}

Authors highly appreciate Universiti Teknologi PETRONAS for the provision of great research facilities.

\section{REFERENCES}

[1] A. Worthington, "On the forms assumed by drops of liquids falling vertically on a horizontal plate," in Proc. the Royal Society of London, 1876, vol. 25, pp. 261-272.

[2] Q. Zhou et al., "Liquid drop impact on solid surface with application to water drop erosion on turbine blades, Part II: Axisymmetric solution and erosion analysis," International Journal of Mechanical Sciences, vol. 50, pp. 1543-1558, 2008.

[3] M. Abuku, H. Janssen, J. Poesen, and S. Roels, "Impact, absorption and evaporation of raindrops on building facades," Building and Environment, vol. 44, pp. 113-124, 2009.

[4] K. S. Lee, C. Y. Cheah, R. J. Copleston, V. M. Starov, and K. Sefiane, "Spreading and evaporation of sessile droplets: Universal behaviour in the case of complete wetting," Colloids and Surfaces a: Physicochemical and Engineering Aspects, vol. 323, pp. 63-72, 2008.

[5] T. D. Blake, "The physics of moving wetting lines," Journal of colloid and Interface Science, vol. 299, pp. 1-13, 2006.

[6] M. Marengo, C. Antonini, I. V. Roisman, and C. Tropea, "Drop collisions with simple and complex surfaces," Current Opinion in Colloid and Interface Science, 2011.

[7] A. S. Moita and A. Moreira, "The deformation of single droplets impacting onto a flat surface," Journal of Fuels Lubricants, pp. 1477-1489, 2002.

[8] R. R. Rioboo, M. M. Marengo, and C. T. Tropea, "Time evolution of liquid drop impact onto solid, dry surfaces," Experiments in Fluids, vol. 33 , pp. 112-124, 2002

[9] I. V. Roisman, R. Rioboo, and C. Tropea, "Normal impact of a liquid drop on a dry surface: model for spreading and receding," in Proc. the Royal Society of London. Series A: Mathematical, Physical and Engineering Sciences, 2002, vol. 458, pp. 1411-1430.

[10] P. A. Lin and A. Ortega, "The Influence of Surface Tension and Equilibrium Contact Angle on the Spreading and Receding of Water Droplets Impacting a Solid Surface," in Proc. the 13th IEEE Intersociety Conference on Thermal and Thermomechanical Phenomena in Electronic Systems, New York, 2012, pp. 1379-1386.

[11] P. A. Lin and A. Ortega, "The influence of surface tension and equilibrium contact angle on the spreading and receding of water droplets impacting a solid surface," pp. 1379-1386, 2012.

[12] Y. Gu and D. Li, "Liquid drop spreading on solid surfaces at low impact speeds," Colloids and Surfaces a: Physicochemical and Engineering Aspects, vol. 163, pp. 239-245, 2000.

[13] K. L. Mittal, Contact Angle, Wettability and Adhesion: Brill Academic Pub, 2009 .

[14] V. Starov, S. Zhdanov, S. Kosvintsev, V. Sobolev, and M. Velarde, "Spreading of liquid drops over porous substrates," Advances in Colloid and Interface Science, vol. 104, pp. 123-158, 2003.

[15] N. C. Reis, R. F. Griffiths, and J. M. Santos, "Parametric study of liquid droplets impinging on porous surfaces," Applied Mathematical Modelling, vol. 32, pp. 341-361, 2008.

[16] N. Alleborn and H. Raszillier, "Spreading and sorption of a droplet on a porous substrate," Chemical Engineering Science, vol. 59, pp. 2071-2088, 2004.

[17] R. Rioboo, C. Bauthier, J. Conti, M. Voué, and J. D. Coninck, "Experimental investigation of splash and crown formation during single drop impact on wetted surfaces," Experiments in Fluids, vol. 35, pp. 648-652, 2003.

[18] A. F. Stalder et al., "Low-bond axisymmetric drop shape analysis for surface tension and contact angle measurements of sessile drops," Colloids and Surfaces a: Physicochemical and Engineering Aspects, vol. 364, pp. 72-81, 2010. 
[19] P. Attané, F. Girard, and V. Morin, "An energy balance approach of the dynamics of drop impact on a solid surface," Physics of Fluids, vol. 19, pp. 012-101, 2007.

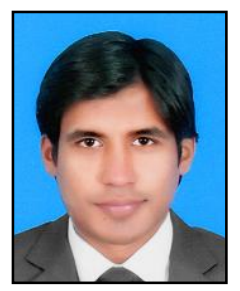

Abdul Basit was born in November, 1985 in Lahore, Pakistan. He received his degree in M. Sc. Engineering (Chemical Engineering) from University of the Punjab Lahore, Pakistan in 2012. He served at the Institute of Chemical Engineering and Technology, University of the Punjab, Lahore, Pakistan as Lecturer from September, 2009 to May, 2012. He is currently studying as a Ph.D. candidate in Universiti Technologi PETRONAS in Department of Chemical Engineering under ONEBAJA project for the production and utilization of a new nitrogen based enhanced efficiency fertilizer. His major filed is computational fluid dynamics modeling of 'droplet spreading on porous surfaces'.

Mr. Abdul Basit is a member of Pakistan Engineering Council (PEC) and Pakistan Institute of Chemical Engineers (PIChE).

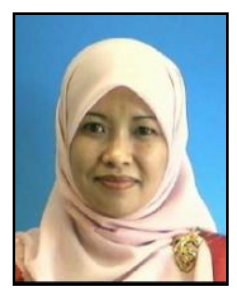

KuZilati KuShaari is an associate professor at the Chemical Engineering Department, Faculty of Engineering, Universiti Teknologi PETRONAS (UTP) and is affiliated to the Chemical Engineering Department as a reaction engineering cluster leader. She holds a degree in Chemical Engineering (Hons.) (University of Detroit Mercy, MI, USA) and Master in Chemical Engineering (West Virginia University, WV, USA). She completed her Ph.D in 2007 at West Virginia University, WV, USA. Her areas of expertise are particle technology, coating and computational fluid dynamic.

She is a member of BEM, IEM, IChemE and AIChE. She was an internship coordinator at UTP for 3.5-years' and has 6 years industrial experience as an engineer. She is also active in research and is currently a project leader of Long Term Research Grant Scheme. She has secured more than RM3 millions research grants, presented in more than 20 international conferences and published her research work in more than 30 indexed journals.

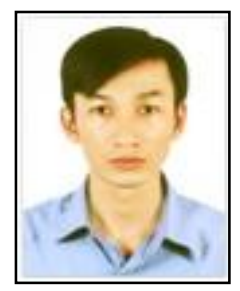

Thanh H. Trinh was born in Ho Chi Minh city, Vietnam on May 28, 1981. He graduated bachelor of Engineering in Chemical Engineering on 2004, and Master in Chemical Engineering on 2007 in Ho Chi Minh City University of Technology (HCMUT), Vietnam. Major fields: processes and units operations, simulation, and analytical chemistry. He started his work in HCMUT from 2004 in courses of processes and units operations, optimization, modeling and simulation. Besides, he cooperated with Agilent and Bruker companies in Vietnam as a customer support, responsible in chromatography applications and FT-IR and FT-NIR applications. From 2010 to 2011, he also worked as an application manager in Key Laboratory of Chemical Engineering and Petroleum Processing - HCMUT. From 2011 until present, study as a PhD candidate in University of Technology PETRONAS in ONEBAJA project for a new nitrogen based enhanced efficiency fertilizer production and utilization.

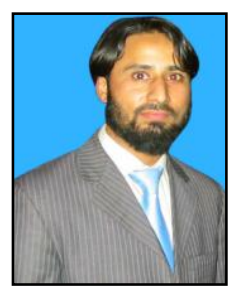

Babar Azeem born in Gujranwala-Pakistan on Feb. 15, 1982. Did his BS-Engg. (Chemical) and MS-Engg. (Chemical) from the most prestigious seat of learning in Pakistan, the University of the Punjab, Lahore, in 2006 and 2011 respectively. Accomplished research projects focused mainly on environmental pollution control. He enjoyed the privilege and honor of working as a research officer and a lecturer at the esteemed University of the Punjab, Lahore-Pakistan for a period of more than 5 years. He also had been the coordinator for Skills Development Program of the University of the Punjab, aimed to train the engineers to have better Professional skills. Currently he is doing his Ph.D. from the Department of Chemical Engineering, Universiti Teknologi Petronas, Malaysia. The axis of his research interests (past \& present) is the Process Economy and Environmental Pollution Control.

Engr. Azeem has professional affiliation with the Pakistan Engineering Council (PEC) and Pakistan Institute of Chemical Engineers (PIChE). He had been an active member of various technical committees to organize the Symposia and Conferences on Engineering Sciences at the University of the Punjab, Lahore-Pakistan. He was awarded the Best Performance Award from the honorable Vice Chancellor, in lieu of his services at the Skills Development Centre of the University of the Punjab, Lahore-Pakistan. 